\title{
The Micro-pits Image Design Method with Direction-uniformity Distribution Based on Minimum Interval Area Scrambling
}

\author{
Hongcai Wang ${ }^{1, ~ a}$ and Yang Wang ${ }^{2, b}$ \\ ${ }^{1}$ Key Laboratory of Mechanics in Advanced Manufacturing, Institute of Mechanics, Chinese \\ Academy of Sciences, Beijing, 100190, China \\ ${ }^{2}$ School of Mechatronics Engineering, Harbin Institute of Technology, Harbin, Heilongjiang, 150001, \\ China \\ ahcwang@imech.ac.cn, bwyyh@hit.edu.cn
}

Keywords: Surface roughness evaluation, Image design method, Uniformity.

\begin{abstract}
It is bigger that $S R a_{d c v}$ of the micro-pits image based on square area scrambling, and the uniformity of micro-pits distribution is not very good. An improved method is presented that presetting the minimum interval of the micro-pits, and setting positions of these micro-pits randomly, then optimizing the positions of the micro-pits by area scrambling. $S R a_{d c v}$ of the micro-pits image generated in this method will be much smaller, and the uniformity of the micro-pits distribution will be very good.
\end{abstract}

\section{Introduction}

Steel plate is the most widely used steel products. Texturing sheet, a kind of steel plate, has excellent stamping performance and coating properties due to its special surface morphology. LT steel plate has better stamping performance and coating properties than SBT and EDT, because the micro-pits have special surface morphology of isolating each other. However, the regular distribution property makes LT steel plate worse in elongation and appearance quality. To overcome the disadvantages and improve the comprehensive performance of the LT sheet, it is required to achieve approximate isotropic and irregular micro-pits distribution.

Problem description of isotropic distribution of micro-pits is how to design the positions of micro-pits whose number is appointed and whose positions should be no overlap in an appointed plane to make $S R a_{d c v}$ the approximate minimum. $S R a_{d c v}$ referred to here is the coefficient of variation of directional surface arithmetic mean deviation in assessed area ${ }^{[1]}$.

This is a quite complex global optimization problem. The number of possible micro-pits images is very large, but is limited afer all. So there must be an optimal image.References [2] raised two image generating algorithms based on square area scrambling, including area scrambling image generating algorithm based on square-aligned-array arrangement and area scrambling image generating algorithm based on square-staggered-array arrangement. $S R a$ in the former algorithm has two minimum values in the directions of 90 degree and 180 degree, while $S R a$ in the latter algorithm has one minimum value in the direction of 0 degree. These minimum values cause the value of $S R a_{d c v}$ bigger. If we set minimum interval of micro-pits according to covering percentage, and confirm positions of micro-pits randomly, $S R a$ of the acquired minimum interval micro-pits image has no obvious dividing line, and $S R a_{d c v}$ will be smaller. $S R a_{d c v}$ of the minimum interval area scrambling micro-pits image will be much smaller.

\section{Image based on minimum interval of micro-pits}

The sketch of a micro-pit is shown in Fig.1. A micro-pit consists of a ring-shaped lug and the pit. In the sectional view, the lug is simulated by the first half cycle of a sine wave, and the pit is simulated by the next half cycle of a sine wave. The external diameter of the micro-pit is $150 \mu \mathrm{m}$ and internal diameter ( the external diameter of the pit) is $100 \mu \mathrm{m}$. The lug height of the micro-pit is $5 \mu \mathrm{m}$. The 
depth of the micro-pit is $20 \mu \mathrm{m}$. In the platform of the micro-pit, the pixel gray value of the plane part is 200 ( This represents the value of the height is $20 \mu \mathrm{m}$ ). The pixel gray value of the top of the lug is 250 ( This represents the value of the height is $25 \mu \mathrm{m}$ ). The pixel gray value of the bottom of the pit is 0 ( This represents the value of the height is $0 \mu \mathrm{m}$ ). The lighter the colour, the higher the height.

The design coordinate system of micro-pits image is shown in Fig.2. The coordinate unit in the figure is the number of pixels ( All the length units without indicating thereinafter are the number of pixels). $1501 \times 1501$ area repesents the size of the micro-pits to generate. The disk whose diameter is 75 represents the size of a micro-pit. The disk in the middle whose diameter is 1175 represents assessed area.

The random search process of the image based on minimum interval of micro-pits (The minimum interval is set to $174 \mu \mathrm{m}$ ) is shown in Fig.3. Different seed usually generates different micro-pits image, and corresponding $S R a_{d c v}$ is also different. After repeated searches, the best approximate isotropic distribution of micro-pits image is obtained. There are totally 194 micro-pits. The result is shown in Fig.4. Its graph of $S R a$ is shown in Fig.5. Its directional accessed indexes of $S R a$ are shown in Table 1.

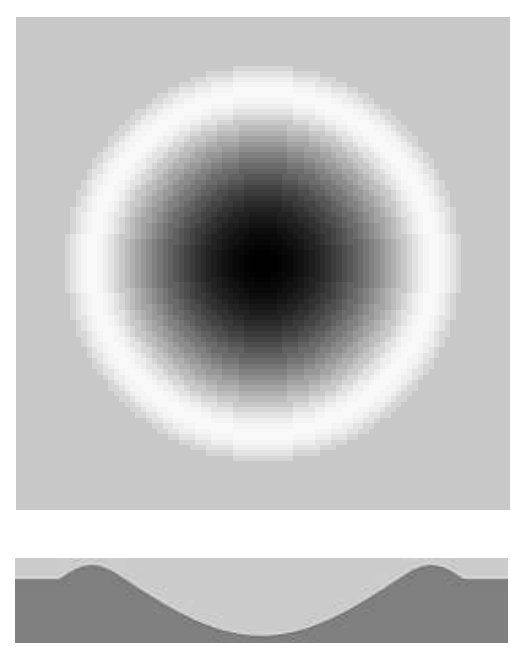

Fig.1 Sketch of a micro-pit

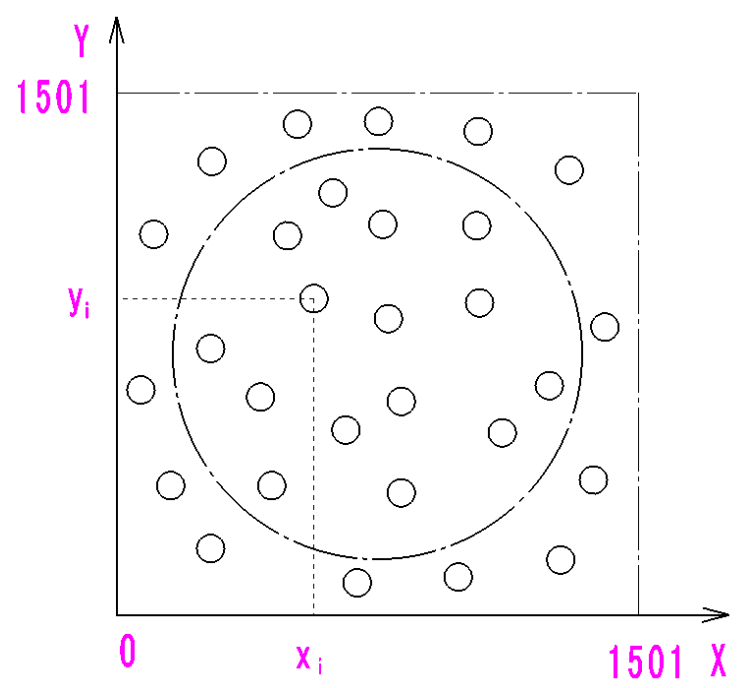

Fig.2 Design coordinate system of micro-pits images

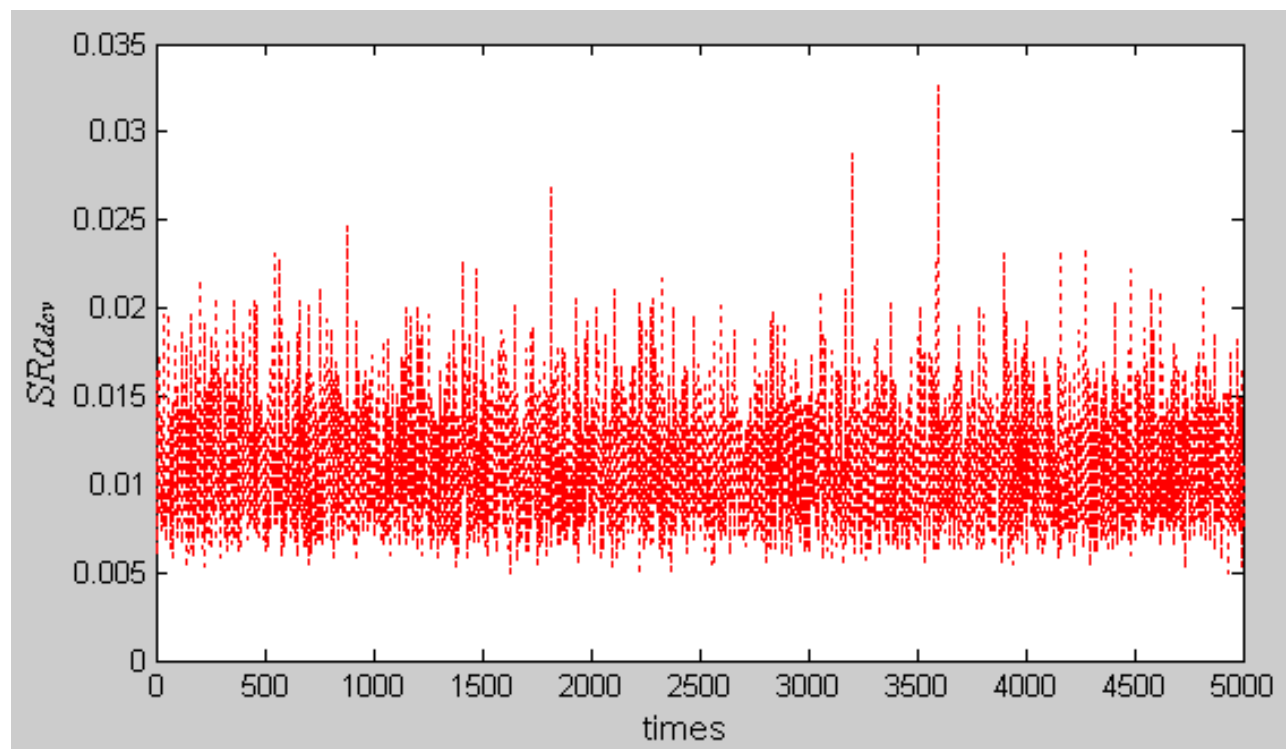

Fig.3 Random search process of the image based on minimum interval of micro-pits 
Table1 Assessed direction indexes of $S R a$ of the image based on minimum interval of micro-pits

\begin{tabular}{cccccc}
\hline$S R a_{\text {dmin }}(\mu \mathrm{m})$ & $S R a_{\text {dmax }}(\mu \mathrm{m})$ & $S R a_{\text {drange }}(\mu \mathrm{m})$ & $S R a_{\text {dmean }}(\mu \mathrm{m})$ & $S R a_{\text {dstd }}(\mu \mathrm{m})$ & $S R a_{\text {dcv }}(\%)$ \\
\hline 2.9288 & 2.9885 & 0.0597 & 2.9554 & 0.0141 & 0.4764 \\
\hline
\end{tabular}

\section{Image based on minimum interval and area scrambling of micro-pits}

The center of each micro-pit is changed in the disk whose diameter is $24 \mu \mathrm{m}$. The optimization target is to make $S R a_{d c v}$ minimum and the adopted algorithms is LDSE (Low Dimensional Simplex Evolution Algorithms) ${ }^{[3]}$. Searching process is shown as Fig.6. As shown in the figure, the optimization is tend to convergence. After repeated searches, the approximate isotropic distribution of micro-pits image is obtained. The result is shown in Fig7. Its graph of $S R a$ is shown in Fig8. Its directional accessed indexes of $S R a$ are shown in Table 2.

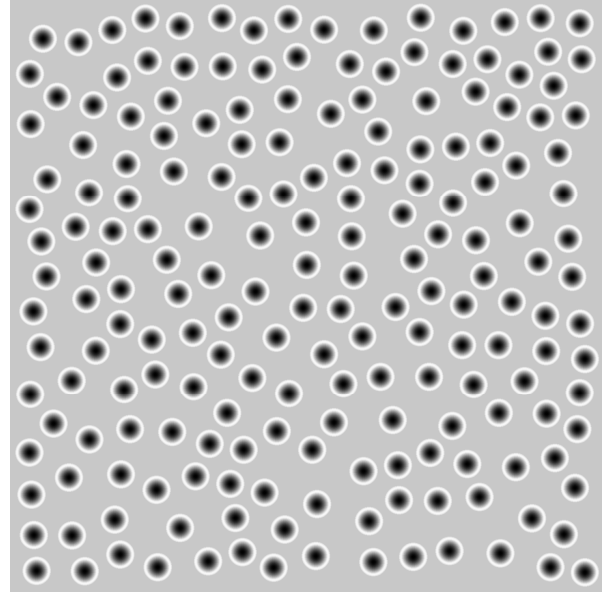

Fig.4 Micro-pits image based on minimum interval

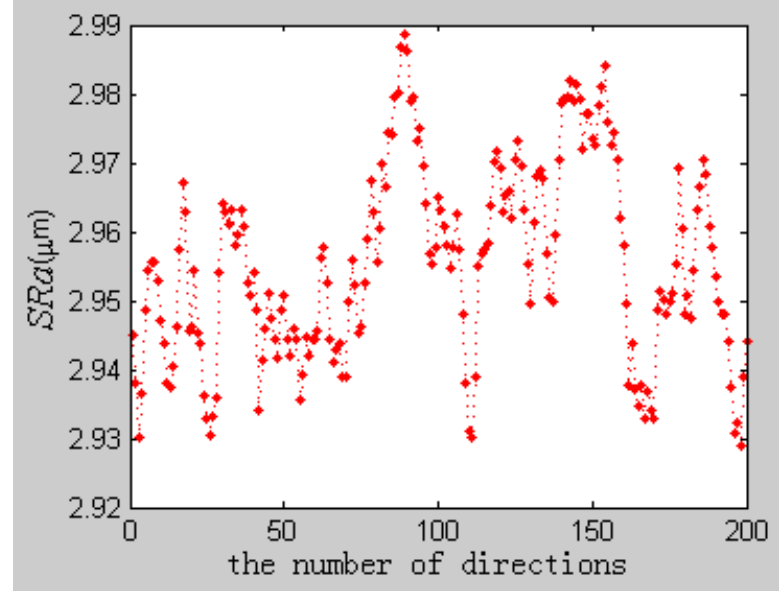

Fig.5 Graph of $S R a$ of the micro-pits image based on minimum interval

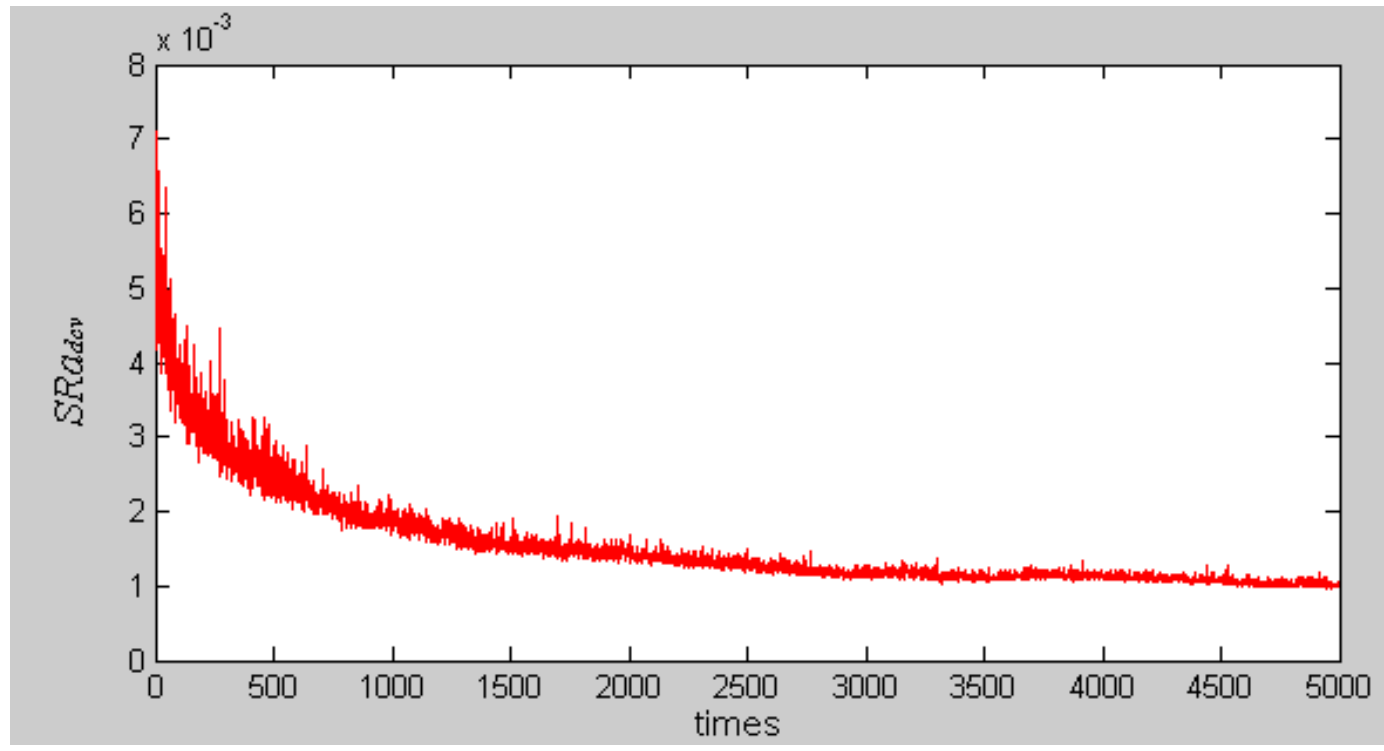

Fig.6 Search process of the image based on minimum interval and area scrambling of micro-pits 
Table2 Assessed direction indexes of SRa of the image based on minimum interval and area scrambling of micro-pits

\begin{tabular}{cccccc}
\hline$S R a_{\text {dmin }}(\mu \mathrm{m})$ & $S R a_{\text {dmax }}(\mu \mathrm{m})$ & $S R a_{\text {drange }}(\mu \mathrm{m})$ & $S R a_{\text {dmean }}(\mu \mathrm{m})$ & $S R a_{d s t d}(\mu \mathrm{m})$ & $S R a_{d c v}(\%)$ \\
\hline 2.9229 & 2.9394 & 0.0165 & 2.9315 & 0.0028 & 0.0967 \\
\hline
\end{tabular}

Compared micro-pits image based on minimum interval and area scrambling with micro-pits image based on minimum interval, $S R a_{d c v}$ reduces $79.7 \%$. The effect is improved significantly.

In references[2], the $S R a_{d c v}$ of square area scrambling micro-pits image based on square-alignedarray arrangement is $0.62 \%$, the $S R a_{d c v}$ of square area scrambling micro-pits image based on squarestaggered-array arrangement is $0.32 \%$. Compared micro-pits image based on minimum interval and area scrambling with these two micro-pits images, $S R a_{d c v}$ reduces $84.4 \%$ and $69.8 \%$. The effect is very good.

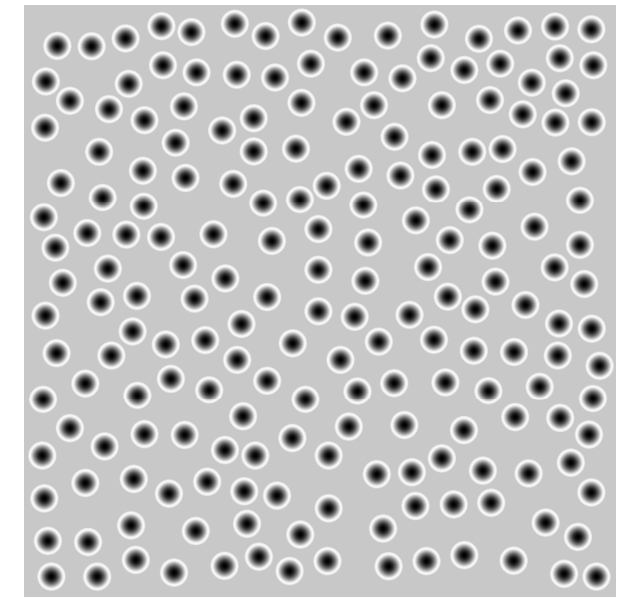

Fig.7 Image based on minimum interval and area scrambling of micro-pits

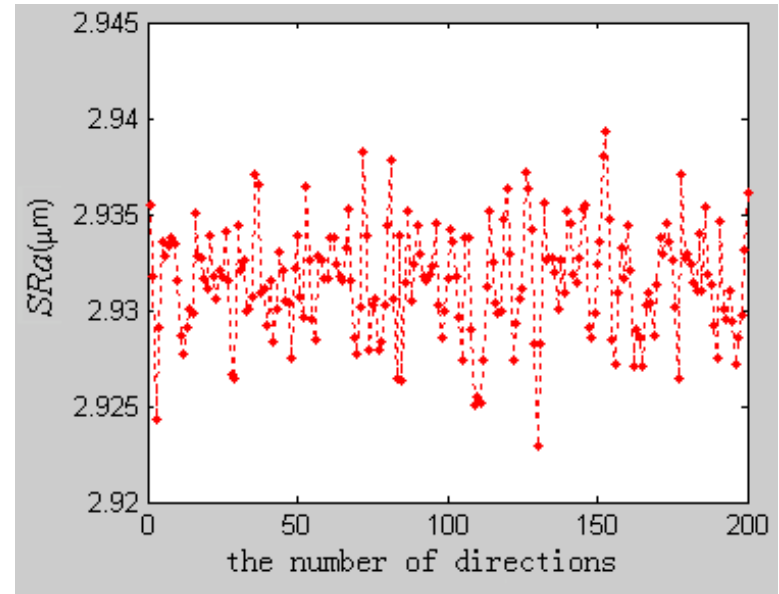

Fig..8 Graph of $S R a$ of the image based on minimum interval and area scrambling of micro-pits

\section{Conclusions}

Comparing with square area scrambling micro-pits images, micro-pits image based on minimum interval and area scrambling has no obvious dividing line among micro-pits, and its $S R a_{d c v}$ is much smaller. So the image design method of micro-pits based on minimum interval and area scrambling is a better method.

\section{References}

[1] Hongcai Wang, Yang Wang. The Height Amplitudes of Surface Roughness based on Area-direction Character and Relative Assessed Indexes[J]. Advanced Material Research, 482-484(2012): 1150-1154.

[2] Hongcai Wang, Yang Wang. The Image Generating Algorithms of Micro-pits with Direction-uniformity Distribution of YAG Laser Texturing Roll Based on Square Partial Scrambling[J]. Advanced Material Research, 538-541(2012): 1908-1912.

[3] Changtong Luo. Low dimensional simplex evolution algorithms and their applications[D]. Jilin University, 2007(in Chinese). 
Mechatronics and Intelligent Materials III

10.4028/www.scientific.net/AMR.706-708

The Micro-Pits Image Design Method with Direction-Uniformity Distribution Based on Minimum Interval Area Scrambling

10.4028/www.scientific.net/AMR.706-708.412 